\title{
Endothelial contractile cytoskeleton and microvascular permeability
}

REVIEW

This article was published in the following Dove Press journal:

Cell Health and Cytoskeleton

8 July 2009

Number of times this article has been viewed

\author{
Qiang Shen \\ Mack HWu \\ Sarah Y Yuan \\ Division of Research, Department \\ of Surgery, University of California \\ at Davis School of Medicine, \\ Sacramento, CA, USA
}

\begin{abstract}
Microvascular barrier dysfunction represents a significant problem in clinical conditions associated with trauma, burn, sepsis, acute respiratory distress syndrome, ischemia-reperfusion injury, and diabetic retinopathy. An important cellular mechanism underlying microvascular leakage is the generation of contractile force from the endothelial cytoskeleton, which counteracts cell-cell and cell-matrix adhesions leading to paracellular hyperpermeability. In this review, we present recent experimental evidence supporting the critical role of MLCK-activated, RhoA/ ROCK-regulated contractile cytoskeleton in endothelial permeability response to inflammatory and thrombotic stimuli arising from thermal injury, activated neutrophils, vascular endothelial growth factor, and fibrinogen degradation products. Further understanding the molecular basis of microvascular barrier structure and function would contribute to the development of novel therapeutic targets for treating circulatory disorders and vascular injury.
\end{abstract}

Keywords: endothelial cell, contractile cytoskeleton, microvascular permeability, signal transduction

\section{Introduction}

The semi-permeable endothelial barrier lining the inner surface of capillaries and venules controls blood-tissue exchange of fluids, electrolytes, and proteins. The integrity of this barrier is crucial in maintaining circulatory homeostasis and the physiological function of different organs. Endothelial hyperpermeability and resulting microvessel leakage are implicated in the development of a variety of disease processes including complications of trauma, hemorrhagic shock, diabetes mellitus, inflammatory disease, myocardial infarction, thrombosis, and tumorigenesis. Through orchestrated molecular events, fluid and molecules move across the endothelial barrier via a transcellular or paracellular pathway. The transcellular pathway is generally responsible for the transport of plasma proteins $(>3 \mathrm{~nm} \mathrm{M})$, such as albumin, by caveolae and vesiculo-vacuolar organelles (VVOs). ${ }^{1,2}$ Small molecules $\left(<3 \mathrm{~nm} \mathrm{M}_{\mathrm{r}}\right.$ ), such as glucose, and plasma fluids are transported through the paracellular pathway that also serves as the major mechanism for transendothelial migration of leukocytes and metastatic tumor cells., ${ }^{2,3}$ The paracellular permeability is dependent on interendothelial junction (IEJ) structures that mediate cell-cell adhesions. To date, two types of endothelial junctions associated with permeability have been characterized: adherens junctions and tight junctions. The tight junctions are formed based on homophilic interactions of occludin, claudins, and junction adhesion molecule (JAM) between the adjacent cells, whereas the adherens junction is mainly composed
Professor and Director of Research, Department of Surgery, University of California Davis School of Medicine, 4625 2nd Avenue, Room 3006, Sacramento, CA 958I7, USA

$\mathrm{Tel}+\mathrm{I}(916) 703-0422$

Fax +I (916) 703-042I

Email sarahyuan@ucdavis.edu 
of VE-cadherin. The intracellular domains of these IEJ structures are connected to the actin cytoskeleton via zona occludin-1 (ZO-1) at tight junctions and catenins $(\alpha, \beta, \gamma$, and p120) at adherens junctions. ${ }^{4,5}$ Furthermore, the endothelial lining is tethered to the extracellular matrix through focal adhesions mediated by transmembrane integrins and a family of actin-linking proteins including focal adhesion kinase, talin, paxillin, and vinculin. ${ }^{6,7}$ These cell-cell and cell-matrix adhesion structures counteract the actomyosin contractile tension thereby maintaining the integrity of vascular endothelium. ${ }^{4,5}$ Imbalance between the adhesive force and contractile force results in barrier dysfunction. Pathophysiological conditions, such as inflammation, trigger a series of signaling events in endothelial cells that propagate to the cytoskeleton and promote cell contraction. Many inflammatory mediators also induce phosphorylation and disorganization of junction molecules. The increased centripetal tension and weakened cell-cell adhesion act in concert leading to intercellular gap formation and paracellular hyperpermeability (Figure 1). 5,8,9

\section{Endothelial contractile machinery}

The endothelial cell cytoskeleton is composed of actin filaments (F-actin), intermediate filaments, and microtubules. Among these filamentous structures, actin is best characterized for its role in regulating microvascular barrier function. Under nonstimulated conditions, the actin cytoskeleton maintains a dynamic equilibrium between polymerization of globular-actin (G-actin) at the barbed end and de-polymerization at the pointed end of F-actin. ${ }^{10}$ The physiological concentration of G-actin is above the critical concentration $\left(\mathrm{C}_{\mathrm{c}}, 0.1 \mu \mathrm{M}\right)$ and favors actin polymerization. ${ }^{10,11}$ Some actin-binding proteins (ABPs), such as thymosin- $\beta$, function to retain this balance by binding to cytosolic free G-actin and preventing it from adding to the barbed end of F-actin. ${ }^{11}$ However, upon activation by an extracellular stimulus, intracellular signaling molecules (eg, phospholipase C) can hydrolyze membrane PtdIns(4,5)P2 and release its associated profilin into the cytosol where it promotes actin polymerization by displacing thymosin- $\beta$ from $G$-actin and facilitating G-actin ADP/ATP exchange. ${ }^{11-13}$ In addition, the PtdIns(4,5)P2-bound gelsolin and cofilin can be released from plasma membrane following phospholipase $\mathrm{C}$ activation ${ }^{13-15}$ and de-polymerize F-actin from the pointed end. Consequently, under the guidance of the Rho family small GTPases ( $\operatorname{Rac} 1, \mathrm{Cdc} 42$, and RhoA), the increased G-actins are polymerized to form cortical lattices, protrusive structures (lamellipodia and filapodia), or stress fibers, which serve different biological functions. ${ }^{16,17}$ Formed beneath the plasma membrane, cortical actin lattices not only control cell shape and stability, but also promote the assembly of cell-cell and cell-matrix adhesions.

The contractile machinery of endothelial cells is mainly driven by a mechanochemical interaction between actin and myosin. As the primary force generator, the activity of myosin is controlled by the phosphorylation status of its regulatory light chain (MLC) (Figure 2). It is well documented that myosin light chain kinase (MLCK) can directly phosphorylate MLC at Thr-18 and/or Ser-19, resulting in a change in the myosin tertiary structure and its sliding movement against actin. ${ }^{18-21}$ As a counter regulator, myosinassociated protein phosphatase (MLCP) dephosphorylates MLC thereby terminating the contraction. ${ }^{22}$ Thus, an optimal control of contraction/relaxation arrives at the MLC phosphorylation level depending on the relative activity of MLCK and MLCP. The Rho family of small GTPases, especially RhoA, acts as a "molecular switch" in this central reaction. ${ }^{23}$ In particular, activation of RhoA (changing from an inactive GDP-bound form to active GTP-bound form) and its downstream kinase ROCK can phosphorylate and inactivate MLCP, thereby promoting MLC phosphorylation and actomyosin contraction. ${ }^{24-26}$ Alternatively, ROCK enhances cell contractility directly by phosphorylating $\mathrm{MLC}^{27}$ or indirectly by activating the $\mathrm{Ca}^{2+}$ /calmodulin-dependent MLCK, ${ }^{9,25,26}$ as the Rho GTPases are capable of stimulating $\mathrm{Ca}^{2+}$ entry into cells. ${ }^{28}$ Recent work also suggests that ROCK is required for VE-cadherin phosphorylation-associated endothelial hyperpermeability. ${ }^{29}$

\section{Endothelial cytoskeletal response and barrier dysfunction}

The MLCK-mediated actomyosin contraction has long been considered a determinant of cell morphology, function, and motility. Over the past decade, many laboratories including ours have suggested a crucial role of MLC phosphorylation in the endothelial permeability response to inflammatory factors, such as thrombin, histamine, oxygen radicals, and leukocytes. ${ }^{20,30-33}$ Using the isolated venule model developed earlier in our laboratory, we have examined the specific effect of MLCK activity on albumin permeability in intact microvessels. ${ }^{31}$ The results show that the MLCK inhibitor ML-7 enhances the basal barrier function and significantly attenuates the agonist-induced hyperpermeability in the coronary system. ${ }^{31}$ In line with this, inhibition of MLC dephosphorylation with calyculin elevates endothelial permeability. ${ }^{31}$ We have further developed a novel 


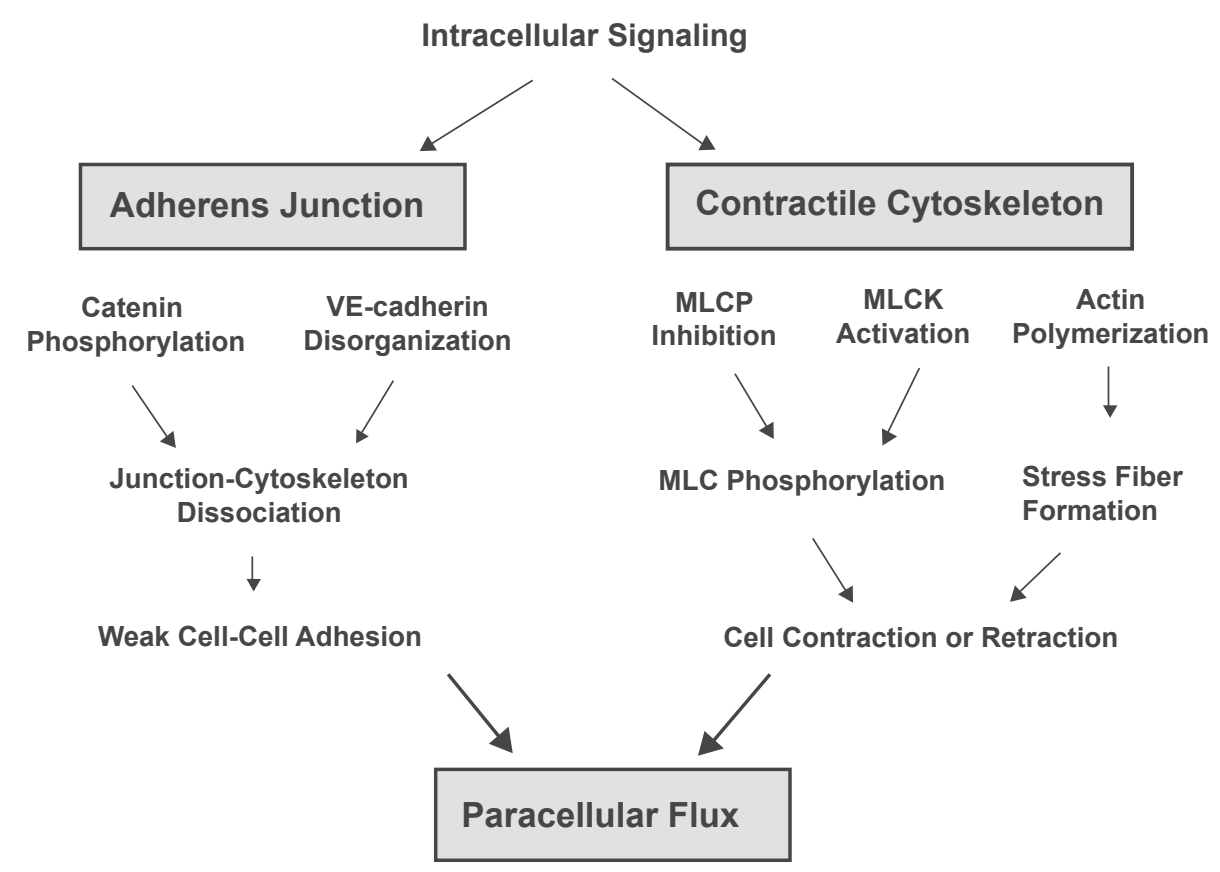

Figure I Endothelial paracellular hyperpermeability. Endothelial paracellular hyperpermeability occurs as a result of orchestrated signaling events at the inter-endothelial junction. The integrity of inter-endothelial junction is maintained by precisely balanced forces generated between intercellular junction structures and actomyosin-based contractile cytoskeleton. Enhanced intracellular signaling, such as $\mathrm{Ca}^{2+}$ and RhoA/ROCK, can promote actin polymerization, activate myosin light chain kinase (MLCK), and inhibit myosin light chain phosphatase (MLCP), thereby inducing MLC phosphorylation and actomyosin contraction. In parallel, phosphorylation of VE-cadherin and catenins ( $\alpha$, $\beta$, $\gamma$, and p 120) causes dissociation of intercellular junction complex from cytoskeletal anchor. As a result, the significantly increased cytoskeleton contractile force and weakened cell-cell adhesion disrupt the balance at intercellular junction, leading to endothelial hyperpermeability.

transfection technique that delivers constitutively active MLCK proteins to the endothelium of intact microvessels as well as to cultured endothelial cell monolayers. ${ }^{34}$ The MLCK-transfected endothelial cells demonstrate a higher level of MLC phosphorylation, accompanied by an enhanced permeability to albumin and contractile morphology characterized by a high density of stress fibers and intercellular gap formation. ${ }^{34}$ Such structural and functional features are diminished after inhibition of MLCK activity, suggesting a causal role for MLCK activation in mediating endothelial cytoskeleton contraction and barrier dysfunction. The following sections further discuss the mechanisms of cytoskeleton-dependent endothelial hyperpermeability under the particular conditions of inflammatory stimulation and thrombotic injury.

\section{MLCK in burn-induced permeability}

Severe trauma and burn typically induce systemic inflammatory response syndrome (SIRS) characterized by microvascular hyperpermeability, tissue edema, hypovolemic shock, and multiple organ dysfunction including acute respiratory distress syndrome (ARDS). ${ }^{35-37}$ We have carried out studies both in vivo and in vitro investigating the endothelial cytoskeleton response and its impact on microvascular permeability in burn-induced inflammation. Exposure of lung microvascular endothelial cell monolayer to plasma harvested from burned animals resulted in approximate 2-fold increase in permeability, a response accompanied by enhanced MLC phosphorylation and stress fiber formation. ${ }^{38}$ Pharmacological inhibitors of MLCK (ML-7) and ROCK (Y-27632) blocked the phosphorylation response and restored barrier function without altering the basal permeability. ${ }^{38} \mathrm{~A}$ similar inhibitory effect was observed during molecular manipulation of MLCK activity in cells transfected with MLCK-inhibiting peptides, suggesting that MLCK-mediated MLC phosphorylation is required for the burn plasma-induced lung endothelial hyperpermeability. In vivo studies using intravital microscopy further demonstrate significant plasma leakage in splanchnic microvessels (eg, in the mesentery) following burn; the barrier injury is greatly attenuated in the presence of the MLCK inhibitors. ${ }^{39}$ Interestingly, inhibition of signaling molecules that are generally considered upstream of the inflammatory response, such as the tyrosine kinase Src and protein kinase C, exhibit negligible inhibitory effect on burn-induced microvascular leakage. ${ }^{39}$ This outcome is consistent with clinical reports regarding limited efficacy of antagonizing individual inflammatory pathways in treating SIRS and multiple organ failure. 

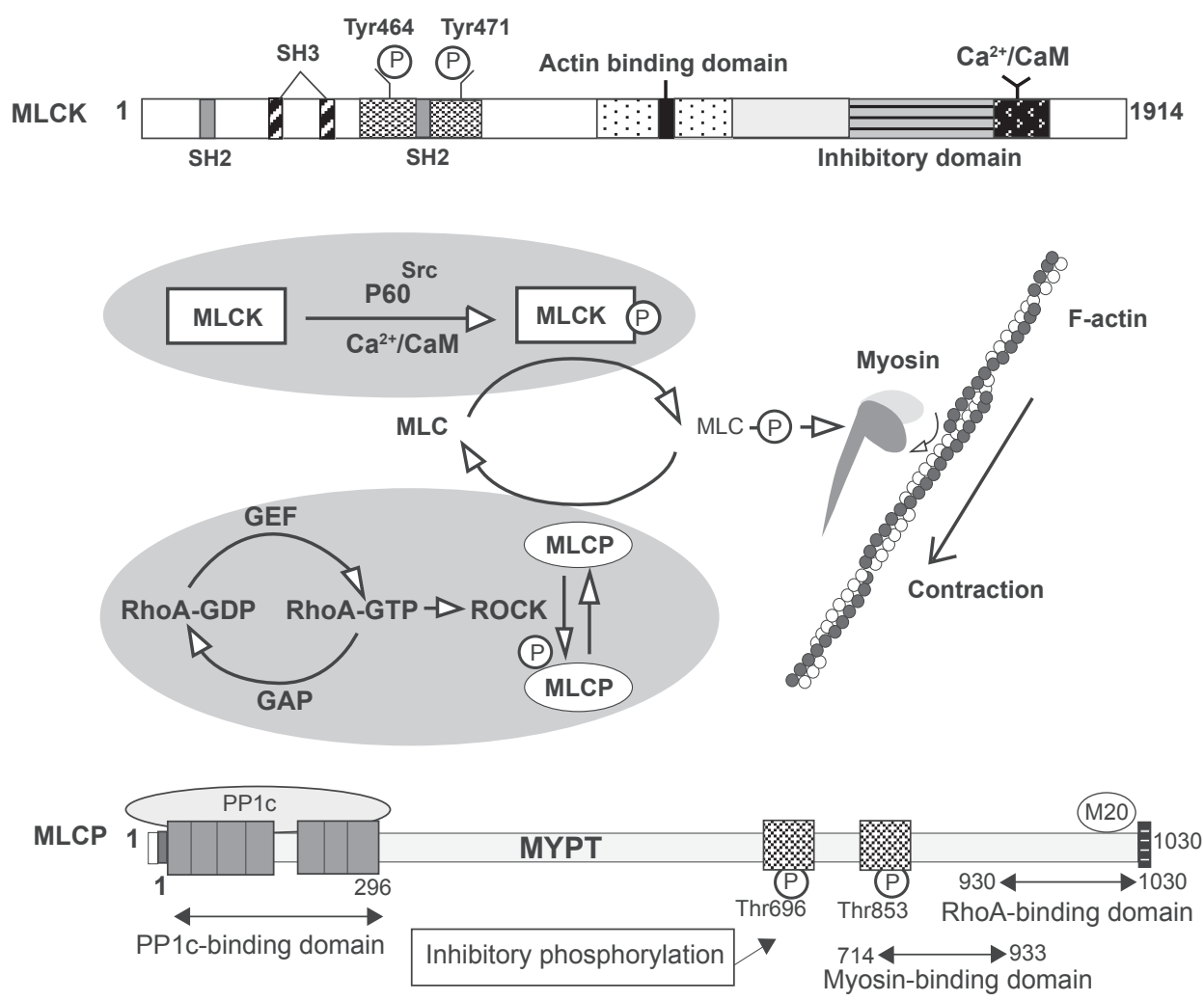

Figure 2 Regulation of MLC phosphorylation. Endothelial cells MLCK consists of N-terminal SH2-/SH3-binding domains, a central actin-binding domain, C-terminal catalytic, and CaM-binding domains. Once MLCK binds to $\mathrm{Ca}^{2+} / \mathrm{CaM}$ and is phosphorylated by tyrosine kinase (eg, $\mathrm{p}^{\mathrm{Src}}$ ) on Tyr-464 and Tyr-47I, it becomes activated and subsequently phosphorylates MLC (Thr-18/Ser-19), resulting in enhanced actomyosin interaction and cell contraction. This MLCK-mediated contractile cytoskeleton can be abrogated by dephosphorylation of MLC via MLC phosphatase (MLCP). MLCP contains a catalytic (PPIc) subunit, a large myosin phosphatase targeting (MYPT) subunit and a 20 kd small subunit. MYPT targets MLCP to the myosin where PPIc subsequently dephosphorylates MLC. In response to RhoA-GTP-mediated activation, ROCK phosphorylates MYPT and thereby disables MLCP to dephosphorylate MLC, leading to actomyosin-based cell contraction.

This is not a surprise considering the wide spectrum of inflammatory mediators ranging from histamine and prostaglandins to leukocyte-derived cytokines that are released during severe trauma. ${ }^{40-42}$ These vasoactive and cytotoxic factors act on the vascular endothelium triggering multiple signaling reactions that crosstalk or interact with each other so inhibition of individual pathway may not be sufficient to block the massive injurious response. On the other hand, developing a therapeutic strategy that specifically targets their common terminal effectors, such as MLCK, may represent a promising direction in burn treatment. Within this context, MLCK has two endothelial isoforms (108 kd and $210 \mathrm{kd}$ ) subjected to various transcriptional processes in different tissues. Among them, the high molecular weight MLCK (MLCK-210) has been recognized as the predominant isoform expressed in vascular endothelial cells. Previous studies have shown that MLCK-210 knockout have relatively low susceptibility to septic injury, especially in the lung. ${ }^{43,44}$ Our recent experiments with these mice show that both albumin extravasation and hydraulic conductivity (Lp) caused by burn are significantly lower in MLCK-210 knockouts than the wild type, and the attenuated microvascular leakage is accompanied by substantially improved survival. ${ }^{45}$ This result extends our previous work ${ }^{31,39}$ specifying the importance of MLCK-210 in the regulation of microvascular permeability in response to inflammation elicited by thermal injury.

\section{MLC phosphorylation in neutrophil- induced permeability}

Endothelial barrier dysfunction is an essential pathophysiological event in the development of cardiovascular disease, such as myocardial infarction, diabetic cardiomyopathy, and atherosclerosis. It has been a common view that endothelial barrier dysfunction is a consequence of leukocyte (eg, neutrophils) activation characterized by adhesion to the endothelium, transmigration, and release of inflammatory mediators. However, the molecular mechanism by which neutrophils cause microvascular hyperpermeability has not been fully understood. We have therefore characterized 
neutrophil-induced signaling in endothelial cells with a focus on the Rho/ROCK- and MLCK-mediated contractile cytoskeleton responses. Perfusion with $\mathrm{C} 5 \mathrm{a}$-activated neutrophils in intact isolated porcine coronary venules induces a time- and concentration-dependent increase in albumin permeability, whereas selective inhibition of MLCK with ML-7 and MLCK-inhibiting peptide abrogates the response. ${ }^{46}$ Furthermore, the hyperpermeability effect of neutrophils can be mimicked by dominant expression of activated MLCK via transfection of constitutively active MLCK into the microvascular endothelium. ${ }^{46}$ Similar results have been achieved from cultured coronary venular endothelial cells where C5a-activated neutrophils induce MLCK-dependent albumin flux. ${ }^{46}$ In an effort to identify the signals upstream from MLC phosphorylation, we tested the potential effects of RhoA and ROCK on the neutrophilinduced microvascular barrier dysfunction. A Rho GTPase pull-down assay indicates that neutrophils trigger timedependent activation of RhoA. Inhibition of ROCK with two structurally distinct pharmacological inhibitors, Y-27632 and HA-1077, substantially attenuates neutrophilinduced albumin flux across endothelial cell monolayers. ${ }^{47}$ In addition, transfection of constitutively active ROCK increases albumin permeability in intact venules and reduces transcellular electric resistance (TER) in endothelial cells cultured in an electrical cell substrate impedance-sensing system (ECIS) ${ }^{48}$ The barrier disruptive effect of ROCK transfection can be inhibited by either Y-27632 or H-1152 in a concentration-dependent manner. ${ }^{48}$ Additional evidence supporting the critical regulatory role of RhoA-ROCK in cytoskeleton-mediated permeability is the finding that hyperpermeability occurs concurrently with an increase in endothelial cell isometric tension..$^{48}$ Moreover, Y-27632 attenuates neutrophil-induced TER decrease and hyperpermeability in isolated coronary venules. ${ }^{48}$ Further molecular analysis with urea gel electrophoresis demonstrates that neutrophils induce di-phosphorylation of MLC at Thr-18/Ser-19 in a concentration- and time-dependent manner. ${ }^{46-48}$ Consistently, immunocytochemistry show that neutrophil stimulation results in significant contractile stress fiber formation in endothelial cells. ${ }^{46-48}$ Both the neutrophil-induced MLC phosphorylation and stress fiber formation are substantially attenuated during inhibition of ROCK with Y-27632 and HA-1077 and inhibition of MLCK with ML-7, ${ }^{46}$ supporting the causal effect of ROCK/MLCK activation and phospho-MLC-mediated cytoskeletal contraction in neutrophil-induced microvascular barrier dysfunction. Collectively, these results suggest that neutrophils activate the ROCK/MLCK signaling in endothelial cells leading to cytoskeleton contraction; this response plays an essential role in neutrophil-induced microvascular hyperpermeability.

\section{RhoA/ROCK in VEGF-induced permeability}

Vascular endothelial growth factor (VEGF) has been well recognized as a critical regulator in various pathophysiological conditions, such as pathological angiogenesis, diabetic retinopathy, ischemia-reperfusion (I-R) injury, and tumor metastasis. All these processes involve microvascular endothelial hyperpermeability. For example, VEGF is shown to exacerbate pulmonary edema and cerebral hemorrhage in animal models of I-R injury. ${ }^{49,50}$ VEGF and it receptor VEGFR-2 are both also upregulated in diabetic retinopathy that is characterized by angiogenesis and microvascular leakage. ${ }^{51}$ Over the past decade, it has been well documented that VEGF can phosphorylate and dissociate IEJ molecules, such as VE-cadherin, thereby promoting endothelial leakage. In addition, we have shown that phospholipase $\mathrm{C}$, PKC, PKG, intracellular $\mathrm{Ca}^{2+}$, MAPK kinase (MEK), and nitric oxide are involved in VEGF-induced vascular barrier dysfunction. ${ }^{52-54}$ in vitro studies provide compelling evidence suggesting that VEGF also activates RhoA and ROCK. ${ }^{55}$ We have subsequently taken a further step to investigate the possible role of RhoA-stimulated contractile cytoskeleton in VEGF-induced endothelial hyperpermeability. In isolated and perfused coronary venules, VEGF $\left(10^{-10} \mathrm{M}\right)$ triggers a rapid but transient increase in albumin permeability; the response peaks at 5 minutes and disappears after 20 minutes of treatment. ${ }^{56}$ This VEGF-induced microvascular hyperpermeability is blocked by the ROCK inhibitor Y-27632 in a dose-dependent manner, with the maximal inhibition seen at $10^{-6}$ M. ${ }^{56}$ Upstream from ROCK, the activation of RhoA is confirmed in a Rho-GTP pull down assay of coronary venular endothelial cells, as VEGF activates RhoA within 1 minute and the effect lasts about 15 minutes. ${ }^{56}$ Likewise, an in vitro Transwell assay demonstrates that the VEGFinduced albumin transendothelial flux is significantly attenuated in cells pretreated with exoenzyme $\mathrm{C} 3$, a bacterial toxin that inactivates RhoA..$^{56}$ The same inhibitors also abrogate VEGF-induced MLC phosphorylation and contractile fiber formation in endothelial cells. ${ }^{56}$ Taken together, these results demonstrate a critical role for RhoA/ROCK signaling in endothelial contractile response to VEGF.

Given the impact of VEGF on many pathogenic processes, tremendous efforts have been devoted to developing 
novel therapies that specifically target the VEGF pathway, including anti-VEGF antibodies, VEGF traps, and VEGF receptor antagonists. Some of these agents have been selected for clinical trials under certain disease conditions. For example, bevacizumab has been shown to inhibit neovascular age-related macular degeneration, tumor growth, and metastasis. ${ }^{57-60}$ In many others cases, however, no substantial efficacies have been achieved with the anti-VEGF strategy. Alternatively, targeting the end-point effectors of VEGF signaling in the endothelium by inhibiting RhoA/ROCK or contractile cytoskeleton may serve as an effective therapy and thus hold promise for future drug development.

\section{Contractile cytoskeleton}

\section{and fibrinogen-induced permeability}

Fibrinogen is a plasma glycoprotein produced by the liver that plays a crucial role in blood coagulation. The plasma level of fibrinogen is considered a risk factor in the development of cardiovascular diseases, such as myocardium infarction, atherosclerosis, stroke, and peripheral vascular disease. ${ }^{61,62}$ Structurally, the fibrinogen is composed of double polypeptides of $\alpha, \beta$, and $\gamma$ chains forming outer globular D domains and central E domains. ${ }^{63-65}$ Following the processes of coagulation cascade and subsequent fibrinolysis, fibrinogen is converted to cross-linked fibrin and then degraded into proteolytic products, including the D-dimer fragments and C-termini of $\alpha, \beta$, and $\gamma$ chains. Emerging evidence suggests that these fibrin degradation products (FDPs) exert biological impacts on cell proliferation, motility, and barrier function. ${ }^{66-69}$ Of particular importance is the $\mathrm{C}$ terminus of fibrinogen $\gamma$-chain $(\gamma \mathrm{C})$, as it contains several reactive regions for fibrin cross-linking and surface receptors binding. Intravital microscopic studies show that administration of $\gamma \mathrm{C}$ at a pathophysiological relevant concentration induces rapid extravasation of plasma albumin and increased hydraulic conductivity in mesenteric microvessels. ${ }^{70}$ In cultured microvascular endothelial cells, $\gamma \mathrm{C}$ induces a time- and dose-dependent reduction in TER, ${ }^{70}$ indicating impaired IEJ adhesion and barrier integrity. Contractile morphology (stress fibers and intercellular gaps) is observed in the cells within the same time course. ${ }^{70}$ Further molecular analyses indicate that the contractile response involves RhoA/ROCK signaling because both RhoA activation and MLC phosphorylation are detected following $\gamma \mathrm{C}$ treatment, and inhibition of ROCK activity or knockdown of RhoA expression is able to block $\gamma \mathrm{C}$ induced contractile morphology and barrier dysfunction. ${ }^{70}$
Thus, endothelial cytoskeleton contraction and resulting paracellular permeability contribute to barrier dysfunction under conditions associated with the production of FDPs. The significance of this response is not limited to explaining coagulation disorders or thrombotic injury, as many pathological processes such as severe burn, inflammation, and myocardial infarction are generally accompanied by FDPs production from the extracellular matrix by activated matrix metalloproteases and leukocyte-derived proteases. Targeting actomyosin-based contractile cytoskeleton may provide a therapeutic tool for the treatment of cardiovascular disease.

\section{Summary}

Microvascular endothelial barrier dysfunction is critical in the development of various diseases. Under physiological conditions, the barrier integrity is maintained based on balanced cell-cell and cell-matrix adhesions against the centripetal tension generated by the contractile cytoskeleton. Many inflammatory mediators or vasoactive agents are capable of inducing or promoting actin-myosin interactions, thereby shifting the balance toward a contractile-dominant conformation that favors intercellular junction opening and paracellular permeability. While our work is focused on the role of MLCK/ROCK in the contractile cytoskeleton-mediated endothelial hyperpermeability in response to inflammation, burn, pathological angiogenesis, and thrombotic injury, we recognize the importance of many other cellular structures and signaling pathways in the regulation of vascular permeability under different physiological and pathological conditions. As examples, the therapeutic potential of the ROCK inhibitor Y-27632 has recently been demonstrated in treating lung injury and ARDS. ${ }^{71,72}$ Another ROCK inhibitor, fasudil, has been evaluated for its impact on cardiovascular protection including an effect in delaying the progress of diabetic retinopathy and nephropathy. ${ }^{73,74}$ Therefore, further studies shall be directed toward a more extensive search for structural elements or signaling molecules that play key roles in the hyperpermeability response. Advanced knowledge in this area may lead to the development of new diagnostic tools or therapeutic targets for improved treatment of vascular disease.

\section{Acknowledgments}

We acknowledge Chris Pivetti and Olesya Litovka for their work in the manuscript preparation. This work is supported by the NIH grants HL61507, HL70752, HL73324, and HL84542. 


\section{References}

1. Feng D, Nagy JA, Hipp J, et al. Vesiculo-vacuolar organelles and the regulation of venule permeability to macromolecules by vascular permeability factor, histamine, and serotonin. J Exp Med. 1996;183: 1981-1986.

2. Mehta D, Malik AB. Signaling mechanisms regulating endothelial permeability. Physiol Rev. 2006;86:279-367.

3. Lewalle JM. Alteration of interendothelial adherens junctions following tumor cell-endothelial cell interaction in vitro. Exp Cell Res. 1997;237:347-356.

4. Iyer S, Ferreri DM, DeCocco NC, et al. VE-cadherin-p120 interaction is required for maintenance of endothelial barrier function. Am J Physiol Lung Cell Mol Physiol. 2004;286:L1143-L1153.

5. Dudek SM, Garcia JG. Cytoskeletal regulation of pulmonary vascular permeability. J Appl Physiol. 2001;91:1487-1500.

6. $\mathrm{Wu} \mathrm{MH}$. Endothelial focal adhesions and barrier function. $J$ Physiol. 2005;569:359-366.

7. Playford MP, Schaller, MD. The interplay between Src and integrins in normal and tumor biology. Oncogene. 2004;23:7928-7946.

8. Prasain N, Stevens T. The actin cytoskeleton in endothelial cell phenotypes. Microvasc Res. 2009;77:53-63.

9. Tiruppathi C, Ahmmed GU, Vogel SM, et al. $\mathrm{Ca}^{2+}$ signaling, TRP channels, and endothelial permeability. Microcirculation. 2006;13: 693-708.

10. Carlier MF, Pantaloni D. Control of actin dynamics in cell motility. J Mol Biol. 1997;269:459-467.

11. Bindschadler M, McGrath JL. Relationships between actin regulatory mechanisms and measurable state variables. Ann Biomed Eng. 2007;35:995-1011.

12. Perelroizen I, Didry D, Christensen H, et al. Role of nucleotide exchange and hydrolysis in the function of profilin in action assembly. J Biol Chem. 1996;271:12302-12309.

13. Goldschmidt-Clermont PJ, Kim JW, Machesky LM, et al. Regulation of phospholipase C-gamma 1 by profilin and tyrosine phosphorylation. Science. 1991;251:1231-1233.

14. Yu FX, Sun HQ, Janmey PA, et al. Identification of a polyphosphoinositide-binding sequence in an actin monomerbinding domain of gelsolin. J Biol Chem. 1992;267:14616-14621.

15. van Rheenen J. EGF-induced PIP2 hydrolysis releases and activates cofilin locally in carcinoma cells. $J$ Cell Biol. 2007;179:1247-1259.

16. Welch MD, Mullins RD. Cellular control of actin nucleation. Annu Rev Cell Dev Biol. 2002;18:247-288.

17. Raftopoulou M, Hall A. Cell migration: Rho GTPases lead the way. Dev Biol. 2004;265:23-32.

18. Garcia JG, Davis HW, Patterson CE. Regulation of endothelial cell gap formation and barrier dysfunction: role of myosin light chain phosphorylation. J Cell Physiol. 1995;163:510-522.

19. Goeckeler ZM, Wysolmerski RB. Myosin light chain kinase-regulated endothelial cell contraction: the relationship between isometric tension, actin polymerization, and myosin phosphorylation. J Cell Biol. 1995;130: 613-627.

20. Hixenbaugh EA. Stimulated neutrophils induce myosin light chain phosphorylation and isometric tension in endothelial cells. Am J Physiol. 1997;273:H981-H988.

21. Verin AD, Gilbert-McClain LI, Patterson CE, et al. Biochemical regulation of the nonmuscle myosin light chain kinase isoform in bovine endothelium. Am J Respir Cell Mol Biol. 1998;19:767-776.

22. Verin AD, Patterson CE, Day MA, et al. Regulation of endothelial cell gap formation and barrier function by myosin-associated phosphatase activities. Am J Physiol. 1995;269:L99-L108.

23. Hall A. Rho GTPases and the actin cytoskeleton. Science. 1998:279: 509-514.

24. Totsukawa G. Distinct roles of ROCK (Rho-kinase) and MLCK in spatial regulation of MLC phosphorylation for assembly of stress fibers and focal adhesions in 3 T3 fibroblasts. $J$ Cell Biol. 2000;150: 797-806.
25. Essler M. Thrombin inactivates myosin light chain phosphatase via Rho and its target Rho kinase in human endothelial cells. J Biol Chem. 1998;273:21867-21874.

26. Essler M. Mildly oxidized low density lipoprotein induces contraction of human endothelial cells through activation of Rho/Rho kinase and inhibition of myosin light chain phosphatase. J Biol Chem. 1999;274:30361-30364.

27. Amano M. Phosphorylation and activation of myosin by Rho-associated kinase (Rho-kinase). J Biol Chem. 1996;271:20246-20249.

28. Mehta D. RhoA interaction with inositol 1,4,5-trisphosphate receptor and transient receptor potential channel-1 regulates $\mathrm{Ca}^{2+}$ entry. Role in signaling increased endothelial permeability. J Biol Chem. 2003;278:33492-33500

29. Sanchez T. Induction of vascular permeability by the sphingosine-1phosphate receptor-2 (S1P2R) and its downstream effectors ROCK and PTEN. Arterioscler Thromb Vasc Biol. 2007;27:1312-1318.

30. Yuan SY. Protein kinase signaling in the modulation of microvascular permeability. Vascul Pharmacol. 2002;39:213-223.

31. Yuan Y, Huang Q, Wu HM. Myosin light chain phosphorylation: modulation of basal and agonist-stimulated venular permeability. Am J Physiol. 1997;272:H1437-H1443.

32. Moy AB, Shasby SS, Scott BD, et al. The effect of histamine and cyclic adenosine monophosphate on myosin light chain phosphorylation in human umbilical vein endothelial cells. J Clin Invest. 1993;92: $1198-1206$

33. Morel NM, Petruzzo PP, Hechtman HB, et al. Inflammatory agonists that increase microvascular permeability in vivo stimulate cultured pulmonary microvessel endothelial cell contraction. Inflammation. 1990;14:571-583.

34. Tinsley JH, De Lanerolle P, Wilson E, et al. Myosin light chain kinase transference induces myosin light chain activation and endothelial hyperpermeability. Am J Physiol Cell Physiol. 2000;279: C1285-1289.

35. Cioffi WG. What's new in burns and metabolism. J Am Coll Surg. 2001;192:241-254.

36. Lund T, Onarheim H, Reed RK. Pathogenesis of edema formation in burn injuries. World J Surg. 1992;16:2-9.

37. Gibran NS, Heimbach DM. Current status of burn wound pathophysiology. Clin Plast Surg. 2000;27:11-22.

38. Tinsley JH, Teasdale NR, Yuan SY. Myosin light chain phosphorylation and pulmonary endothelial cell hyperpermeability in burns. Am J Physiol Lung Cell Mol Physiol. 2004;286:L841-L847.

39. Huang Q, Xu WJ, Ustinova EE, et al. Myosin light chain kinasedependent microvascular hyperpermeability in thermal injury. Shock. 2003;20:363-368.

40. Gibran NS, Heimbach DM. Mediators in thermal injury. Semin Nephrol. 1993;13:344-358.

41. Kowal-Vern A, Walenga JM, Sharp-Pucci M, et al. Postburn edema and related changes in interleukin-2, leukocytes, platelet activation, endothelin-1, and C1 esterase inhibitor. J Burn Care Rehabil. 1997; 18:99-103.

42. Kurose I, Wolf R, Miyasaka M, et al. Microvascular dysfunction induced by nonsteroidal anti-inflammatory drugs: role of leukocytes. Am J Physiol. 1996;270:G363-G369.

43. Wainwright MS. Protein kinase involved in lung injury susceptibility: evidence from enzyme isoform genetic knockout and in vivo inhibitor treatment. Proc Natl Acad Sci U S A. 2003;100:6233-6238.

44. Behanna HA, Watterson DM, Ranaivo HR. Development of a novel bioavailable inhibitor of the calmodulin-regulated protein kinase MLCK: a lead compound that attenuates vascular leak. Biochim Biophys Acta. 2006; 1763:1266-1274.

45. Reynoso R, Perrin RM, Breslin JW, et al. A role for long chain myosin light chain kinase (MLCK-210) in microvascular hyperpermeability during severe burns. Shock. 2007;28:589-595.

46. Yuan SY, Wu MH, Ustinova EE, et al. Myosin light chain phosphorylation in neutrophil-stimulated coronary microvascular leakage. Circ Res. 2002;90:1214-1221. 
47. Breslin JW, Yuan SY. Involvement of RhoA and Rho kinase in neutrophil-stimulated endothelial hyperpermeability. Am J Physiol Heart Circ Physiol. 2004;286:H1057-H1062.

48. Breslin JW, Sun H, Xu WJ, et al. Involvement of ROCK-mediated endothelial tension development in neutrophil-stimulated microvascular leakage. Am J Physiol Heart Circ Physiol. 2006;290:H741-H750.

49. Abumiya T, Yokota C, Kuge Y, et al. Aggravation of hemorrhagic transformation by early intraarterial infusion of low-dose vascular endothelial growth factor after transient focal cerebral ischemia in rats. Brain Res. 2005;1049:95-103.

50. Godzich M. Activation of the stress protein response prevents the development of pulmonary edema by inhibiting VEGF cell signaling in a model of lung ischemia-reperfusion injury in rats. Faseb $J$. 2006;20:1519-1521.

51. Caldwell RB. Vascular endothelial growth factor and diabetic retinopathy: role of oxidative stress. Curr Drug Targets. 2005;6:511-524.

52. Wu HM, Huang Q, Yuan Y, et al. VEGF induces NO-dependent hyperpermeability in coronary venules. Am J Physiol. 1996;271: H2735-H2739.

53. Wu HM, Yuan Y, Zawieja DC, et al. Role of phospholipase C, protein kinase $\mathrm{C}$, and calcium in VEGF-induced venular hyperpermeability. Am J Physiol. 1999;276:H535-H542.

54. $\mathrm{Wu} \mathrm{MH}$, Yuan SY, Granger HJ. The protein kinase MEK1/2 mediate vascular endothelial growth factor- and histamine-induced hyperpermeability in porcine coronary venules. $J$ Physiol. 2005;563: 95-104.

55. Leung T, Chen XQ, Manser E, et al. The p160 RhoA-binding kinase ROK alpha is a member of a kinase family and is involved in the reorganization of the cytoskeleton. Mol Cell Biol. 1996;16:5313-5327.

56. Sun H, Breslin JW, Zhu J, et al. Rho and ROCK signaling in VEGFinduced microvascular endothelial hyperpermeability. Microcirculation. 2006;13:237-247.

57. Barakat MR, Kaiser PK. VEGF inhibitors for the treatment of neovascular age-related macular degeneration. Expert Opin Investig Drugs. 2009;18:637-646.

58. Lee FY. Synergistic antitumor activity of ixabepilone (BMS-247550) plus bevacizumab in multiple in vivo tumor models. Clin Cancer Res. 2008;14:8123-8131.

59. Gerber HP, Kowalski J, Sherman D, et al. Complete inhibition of rhabdomyosarcoma xenograft growth and neovascularization requires blockade of both tumor and host vascular endothelial growth factor. Cancer Res. 2000;60:6253-6258.
60. Zhang J. Elevated expression of vascular endothelial growth factor correlates with increased angiogenesis and decreased progression-free survival among patients with low-grade neuroendocrine tumors. Cancer. 2007;109:1478-1486.

61. Saigo M, Hsue PY, Waters DD. Role of thrombotic and fibrinolytic factors in acute coronary syndromes. Prog Cardiovasc Dis. 2004;46: 524-538.

62. Zacharowski K, Zacharowski P, Reingruber S, et al. Fibrin(ogen) and its fragments in the pathophysiology and treatment of myocardial infarction. J Mol Med. 2006;84:469-477.

63. Mosesson MW. Fibrinogen and fibrin structure and functions. J Thromb Haemost. 2005;3:1894-1904.

64. Doolittle RF. Determining the crystal structure of fibrinogen. J Thromb Haemost. 2004;2:683-689.

65. Lord ST. Fibrinogen and fibrin: scaffold proteins in hemostasis. Curr Opin Hematol. 2007;14:236-241.

66. Tyagi N, Roberts AM, Dean WL, et al. Fibrinogen induces endothelial cell permeability. Mol Cell Biochem. 2008;307:13-22.

67. Allard MF, Doerschuk CM, Brumwell ML, et al. Oleic acid-induced lung injury in rabbits: effect of fibrinogen depletion with Arvin. J Appl Physiol. 1988;64:920-928.

68. Ge M, Ryan TJ, Lum H, et al. Fibrinogen degradation product fragment D increases endothelial monolayer permeability. Am J Physiol. 1991;261:L283-L289.

69. Lo SK, Del Vecchio PJ, Lum H, et al. Fibrin contact increases endothelial permeability to albumin. J Cell Physiol. 1992;151:63-70.

70. Guo M, Daines D, Tang J, et al. Fibrinogen-gamma C-terminal fragments induce endothelial barrier dysfunction and microvascular leak via integrin-mediated and RhoA-dependent mechanism. Arterioscler Thromb Vasc Biol. 2009;29:394-400.

71. Chiba Y, Ishii Y, Kitamura S, et al. Activation of rho is involved in the mechanism of hydrogen-peroxide-induced lung edema in isolated perfused rabbit lung. Microvasc Res. 2001;62:164-171.

72. Koksel O. Rho-kinase (ROCK-1 and ROCK-2) upregulation in oleic acid-induced lung injury and its restoration by Y-27632. Eur J Pharmacol. 2005;510:135-142.

73. Arita R. Rho kinase inhibition by fasudil ameliorates diabetes-induced microvascular damage. Diabetes. 2009;58:215-226.

74. Peng F. RhoA/Rho-kinase contribute to the pathogenesis of diabetic renal disease. Diabetes. 2008;57:1683-1692.
Cell Health and Cytoskeleton

\section{Publish your work in this journal}

Cell Health and Cytoskeleton is an international, peer-reviewed open access journal focusing on all aspects of cell structure and function contributing to normal physiology and cell health and exploring the pathogenesis of cell dysfunction leading to adverse conditions and disease in the organism. The journal welcomes papers covering original research,

\section{Dovepress}

basic science, reviews and evaluations, guidelines, expert opinion and commentary, case reports and extended reports. The manuscript management system is completely online and includes a very quick and fair peerreview system, which is all easy to use. Visit http://www.dovepress.com/ testimonials.php to read real quotes from published authors. 